\title{
A novel adaptive spatial scissor-hinge structural mechanism for convertible roofs
}

\author{
Yenal Akgün ${ }^{\mathrm{a}, *}$, Charis J. Gantes ${ }^{\mathrm{b}, \mathrm{c}}$, Werner Sobek ${ }^{\mathrm{d}}$, Koray Korkmaz ${ }^{\mathrm{a}}$, Konstantinos Kalochairetis ${ }^{\mathrm{b}, \mathrm{c}}$ \\ a Department of Architecture, Izmir Institute of Technology, Gülbahçe Kampüsü 35430 Izmir, Turkey \\ ${ }^{\mathrm{b}}$ Department of Civil Engineering, National Technical University of Athens, 9, Iroon Polytechneiou Str. GR-15780 Athens, Greece \\ ${ }^{\mathrm{c}}$ Metal Structures Laboratory, Zografou Campus, National Technical University of Athens, 9, Iroon Polytechneiou Str. GR-15780 Athens, Greece \\ ${ }^{\mathrm{d}}$ Institute for Lightweight Structures and Conceptual Design, University of Stuttgart, Pfaffenwaldring 14, 70569 Stuttgart, Germany
}

\section{A R T I C L E I N F O}

\section{Article history:}

Received 28 September 2010

Received in revised form

13 January 2011

Accepted 17 January 2011

Available online 8 February 2011

\section{Keywords:}

Scissor-hinge structural mechanisms

Spatial deployable structures

Kinetic structures

Geometrically nonlinear finite element analysis

\begin{abstract}
A B S T R A C T
In this paper, a new adaptive deployable spatial scissor-hinge structural mechanism (SSM) is introduced, which can be converted by means of actuators between a multitude of arch-like, dome-like and double curved shapes, where it can be stabilized and carry loads. This novel SSM is a spatial extension of a planar SSM introduced recently that can achieve a wide range of planar geometries. Main differences of the proposed structural mechanism from current deployable structures are the new connection type of the primary units and the proposed modified spatial scissor-like element (MS-SLE). With the development of this new connection detail and the modified element, it becomes possible to change the geometry of the whole system without changing the dimensions of the struts or the span. After presenting some disadvantages of current deployable structures and outlining the main differences of the proposed spatial SSM with existing examples, the dimensional properties of the primary elements are introduced. Then, geometric principles and shape limitations of the whole structure are explained. Finally, structural analyses of a typical structure in two different geometric configurations are performed, in order to discuss stiffness limitations associated with the advantage of increased mobility.
\end{abstract}

(C) 2011 Elsevier Ltd. All rights reserved.

\section{Introduction}

A mechanism can be defined as a group of rigid bodies connected to each other by rigid kinematic pairs to transmit force and motion [1]. Most movable, foldable, deployable and convertible structures behave as mechanisms during their conversion process and as a load resisting entity when they are fixed. Thus, they have the characteristics of both a mechanism and a bearing structure. These types of mechanisms are called structural mechanisms [2]. Deployable structures are defined as structures that can be transformed from a closed compact configuration to a predetermined, expanded form, in which they are stable and can carry loads [3]. Most deployable structures are structural mechanisms.

Most of the deployable structures described in the literature have predefined open and closed body forms; and transformations occur only between these two forms by using one of the various available transformation types such as sliding, deploying, and folding [4] (Fig. 1). Thus, although some parts of these structures do move, rotate or slide, the overall shape and the geometric variety of these structures never change. Hence, such structures

\footnotetext{
* Corresponding author. Tel.: +90 5066658168.

E-mail address: yenalakgun@gmail.com (Y. Akgün).
}

are considered insufficient for offering real form flexibility. This deficiency provided the motivation for the present study.

Scissor-hinge systems are one of the most common types of deployable structures. Beginning from Spanish engineer Piñero's works in 1960s, many researchers have studied the development of novel planar and spatial scissor-hinge structural mechanisms (SSMs). By using the simple planar scissor-like element (SLE), Piñero designed reticulated single and double-layer domed and various spatial space grids, such as mobile theaters, pavilions and exhibition buildings. Moreover, he designed the first foldable space grid, in which the covering was attached to the structure during deployment [5]. Following Piñero, Escrig and his colleagues Sanchez and Valcarcel at the School of Architecture in Seville have experimented with lightweight folding spatial grid structures. They mainly focused on development of new spatial grids and patterns for deployable arches, geodesic domes and large scale umbrellas [6-10]. While developing these novel grids, they proposed several connection details, and connection elements for various patterns. Calatrava proposed different deployable mechanisms and spatial grids in his Ph.D. thesis [11], and tried to increase the flexibility and adaptability in his designs [12]. Hoberman is a key figure in the research area of deployable SSMs. Following the development of the angulated element, which is a variation of the simple SLE, he designed various deployable spatial SSMs like Iris Dome or Hoberman Sphere [13]. In addition 

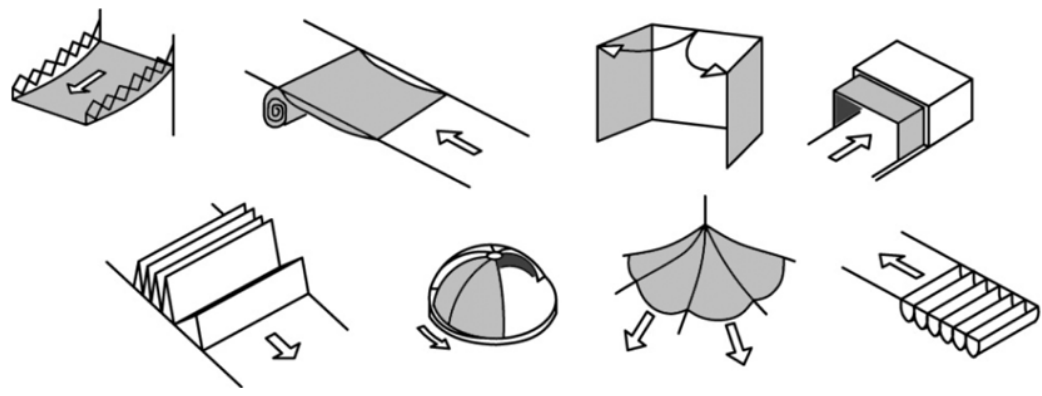

Fig. 1. Examples of transformation types for deployable systems.

to these key researchers and their designs, Al Khayer's polygonal hyperboloids [14] and Diéguez's Florin System [15] are other remarkable examples of innovative designs of SSMs.

Besides the studies on development of new mechanisms, some researchers have focused on explaining the structural, geometric and kinematic behaviors of SSMs by various analytical, numerical and geometrical methods. Escrig exposed the main geometric principles of his designs [16]. Gantes's [3,17-19], Langbecker's [20,21] and Zhao's [22] studies explained the main principles, geometric properties and shape limitations of both planar and spatial SSMs, which were designed by Escrig, Piñero and others. Pellegrino and his research team in Cambridge University improved the design of Hoberman, explained the geometry of structural mechanisms in analytical and numerical ways and proposed several novel concepts [23-25].

When all aforementioned examples are studied thoroughly, it can be seen that their shapes can basically be converted during the opening or closing processes, so that they can only meet specific deployed and contracted configurations. Moreover, all deployable structures in the literature change the size of the covered area during the transformation process. Thus, it can be claimed that existing deployable structures are not adequate to be used as permanent transformable coverings which can achieve multiple shapes without changing the size and boundaries of the covered area. In other words, they do not offer real geometric flexibility.

Aiming to provide a solution to this, the primary objective of the present study is to propose a novel analytical design and implementation framework for spatial deployable structures, which can offer a wide range of form flexibility without changing the size of the covered area. To arrive at this objective, first a novel planar scissor-hinge structure has been proposed in previous work of the authors [26]. This planar structure can achieve a wide range of planar geometries without changing the size of the covered area. In the present work a spatial SSM is being proposed as an extension to the planar SSM. This is not simply an extension of planar SSMs. Instead, a grid of intersecting planar SSMs is proposed, that are appropriately connected to each other, so that their independent motion is possible and arbitrary curvatures can be achieved in both directions. Thus, the proposed spatial SSM can transform its shape between doubly curved geometries and forms, also without changing the size of the covered area.

Hence, the proposed structural mechanism expands the transformation capability, adaptability and form flexibility of deployable structures. Until now, deployable structures have been only used as portative building components. However, after the proposed model of this paper, it is envisaged that deployable structures will also be used as permanent transformable building coverings.

In summary, the most important superiority of the proposed structure over conventional deployable structures is that it can change its shape according to the functional needs, environmental conditions or architectural necessities. A structure which can achieve these transformations can be incorporated as a sustainable

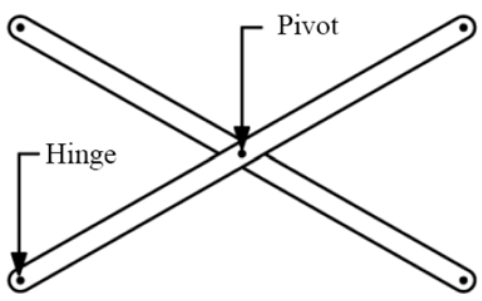

Fig. 2. Scissor-like Element (SLE).

building component. For example, such a transformable structure can be used as a solar roof, attaching solar panels onto its top. Thus, the proposed roof can be rotated according to the location of the sun and benefit from the sun more than conventional solar panels. In another example, the proposed structure can be used as a roof of a sports hall. By changing the height of the roof according to the requirements of the type of sports activity, climatization costs can be decreased. Scissor-hinge structures are conveniently used with control systems, so by using electric motors, these transformations can be performed easily. Use of actuators will slightly increase the construction and operational costs of the roof, but the above mentioned expected benefits make this a small price to pay.

In order to understand the advantages of the proposed spatial SSM, first, general characteristics of scissor-hinge structures are summarized. Then, the proposed SSM, its primary elements, and geometric capabilities are presented. Finally, a series of structural analyses of a typical example of the proposed structures are carried out at two different geometric configurations, and the results are studied, in order to evaluate to what extend increased geometric flexibility is achieved at the expense of reduced stiffness and strength.

\section{Geometric and kinematic properties of common spatial SSMs}

SLE is the primary unit of SSMs. To form an SLE, two bars are connected to each other at an intermediate point through a pivotal connection which allows them to rotate freely about an axis perpendicular to their common plane but restrains all other degrees of freedom (see Fig. 2). At the same time, end points of these bars are hinged to the end points of adjacent scissor units [4].

The transformation properties of the SSMs as well as the orientation of the major axes between the hinge points, which are also the intersection points of the primary SLEs, depend on the location of the pivotal connection. When the pivot point is at the middle point of the struts and the major axes are parallel with each other, the SSMs are called translational (Fig. 3(a)). When the pivotal point of the SLE is not in the middle $\left(a_{1}>b_{1}\right)$, and the major axes intersect at one point, the SSMs are called curved (Fig. 3(b)).

SLEs are planar mechanisms, but they can be used to construct a spatial scissor shell by addition of appropriate connection elements, allowing bars to rotate about axes that are perpendicular to the planes of the corresponding SLEs. An example of contracted 


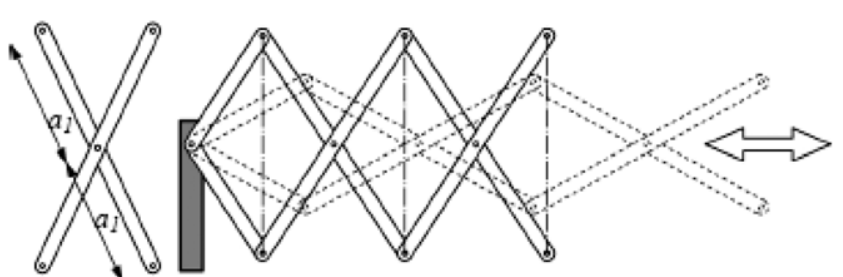

(a) Translational SSM.

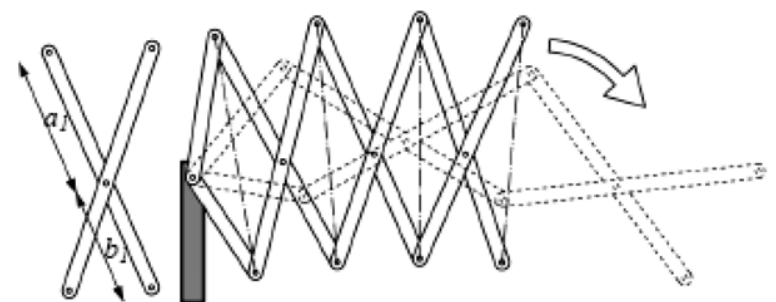

(b) Curved SSM.

Fig. 3. Translational and curved SSMs.

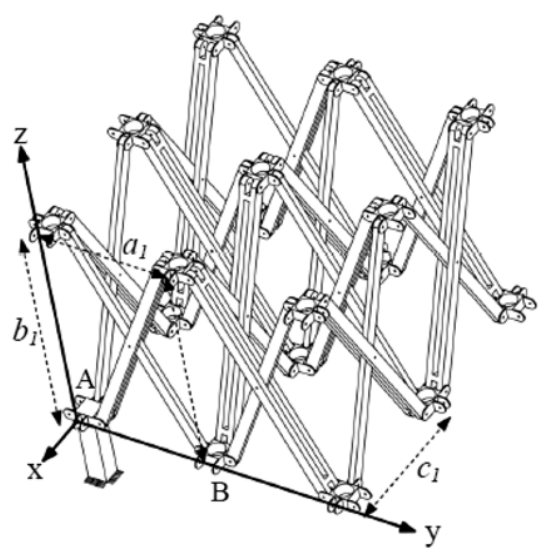

(a) Contracted configuration.

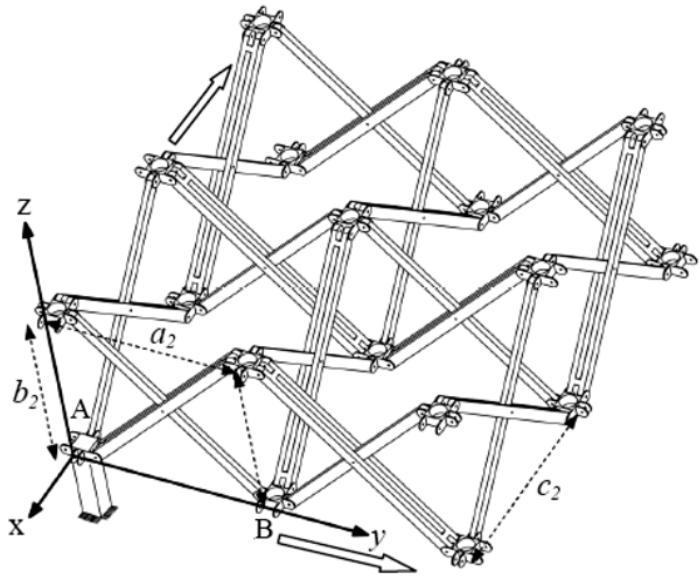

(b) Deployed configuration.

Fig. 4. Transformation of a common spatial translational SSM.

and deployed geometries of a translational spatial SSM is graphically illustrated in Fig. 4. The contracted SSM in Fig. 4(a) is hinged to the ground at $A$, while all other lower nodes are restrained from vertical displacement but are free to slide horizontally. When B is forced to slide along axis $y$ (Fig. 4(b)), all members adapt themselves to this movement. As a result, all distances between nodes change ( $a_{1}<a_{2}, b_{2}<b_{1}, c_{1}<c_{2}$ ). Thus this is an example of spatial SSMs that change their boundaries and the size of the covered area during deployment.

To comprehend the geometric and kinematic characteristics of common deployable structures, the mobility of these structural mechanisms should be investigated as well. A mechanical system's mobility (M) can be classified according to the number of degrees of freedom (DoF) that it possesses. The system's DoF is equal to the number of independent parameters that are needed to uniquely define its position in space at any instant of time [27]. As another definition, mobility is equal to the number of additional actuators or motors needed to control or fix the system. For all transformable and convertible structures, mobility of the system is larger than or equal to one $(M \geq 1)$, and transformation capability increases according to the increase of the mobility value. If the mobility of a kinematic system is equal to zero $(M=0)$, the structure is stable and requires no additional stabilization.

For most common deployable structures, like the example of Fig. 4 , mobility is equal to one $(M=1)$. This means that these structures need only one actuator to move and fix the system. However, $M=1$ also means that these structures can only achieve simple predefined forms. According to simplified version of Freudenstein-Alizade formula [28] the mobility of a system can be calculated as follows:

$M=J_{T}-\lambda L$

where $J_{T}$ includes the contribution of DoFs of all joints, $L$ is the number of loops, $\lambda$ is the number of active DoFs of the space where the mechanism operates $(\lambda=3$ for planar systems and $\lambda=6$ for spatial systems). When this formula is applied to the translational SSM in Fig. 3(a), it can be seen that this mechanism has two loops and eight joints (there are two joints at the support point). $\lambda$ is equal to three for planar mechanisms; so mobility of this system is equal to two $(M=2)$. The curved SSM in Fig. 3(b) has three loops and eleven joints, thus having again a mobility of two $(M=2)$. These results show that both of these SSMs need two actuators to be controlled: one to control rotation around the support and one to control deployment. Mobility of the spatial SSM in Fig. 4 is equal to one $(M=1)$. This means that one actuator is sufficient to transform and fix the mechanism. Most common deployable structures are of the same geometric nature and have mobility equal to one.

\section{Primary elements of the proposed spatial SSM}

The primary elements of the proposed spatial SSM are derived from the previously mentioned planar SLE and the Modified Scissor-Like Element (M-SLE). M-SLE is a planar mechanism that has been developed during the research on planar SSM [1], and it is obtained from the connection of four struts by three hinges on a common point (Fig. 5). This novel element is obtained from the connection of four struts by three hinges on a common point (Fig. 5). Each of the four struts can rotate freely about their common point, without affecting the other three struts. All dimensions of an M-SLE can be random, but according to the geometric analyses [1], it can be seen that the M-SLE gives the most feasible similarity when its dimension is the same as the other SLEs in the system. While the mobility of an SLE is equal to one, the corresponding mobility of an M-SLE is equal to three. In a common planar SSM, when one SLE moves, all other SLEs follow this movement. However, in the proposed SSM, M-SLEs divide the whole system into sub-structures, acting as "isolators", so that each sub-structure 


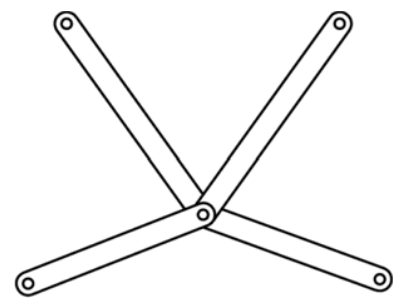

Fig. 5. Modified Scissor-like Element (M-SLE).

can have partial transformation independency without directly affecting the other sub-structures.

For example, the system in Fig. 6 has two M-SLEs, dividing the whole structure into three "isolated" parts. Thus, movement of one SLE in one group is followed only by the SLEs in this group, not by the others. This independency of the sub-groups creates the desired additional geometric mobility; and the whole planar SSM can have form flexibility without changing the span length. Mobility of this system in Fig. 6 is equal to four.

By extending the main principles of the planar M-SLE and the planar SSM, the proposed spatial SSM has been developed. To comprehend the originality and transformational superiority of this spatial SSM, first, primary elements and their geometric capabilities should be thoroughly investigated. The proposed spatial SSMs consist of three types of primary elements (Fig. 7):

- Spatial Scissor-Like Element (S-SLE), consisting of two planar SLEs located in mutually perpendicular planes and connected by appropriate joints, allowing bars to rotate about axes that are perpendicular to the planes of the corresponding SLEs.

- Modified Spatial Scissor-Like Element (MS-SLE), consisting of two planar M-SLEs located in mutually perpendicular planes and connected by appropriate joints, allowing bars to rotate about axes that are perpendicular to the planes of the corresponding M-SLEs.
- Hybrid Spatial Scissor-Like Element (HS-SLE), which is a combination of S-SLE and MS-SLE, consisting of one planar SLEs and one planar M-SLE located in mutually perpendicular planes and connected by appropriate joints, allowing bars to rotate about axes that are perpendicular to the corresponding planes. In Fig. 5, struts which are parts of planar SLEs are shown as white, while those belonging to planar M-SLEs are shown as gray.

To form curvilinear shapes easier, the proposed SSM has been designed as a spatial extension of the planar curved SSM. Thus, all three primary elements of the proposed spatial SSM should be proper to obtain curved planar SLEs and M-SLEs, and length $a$ should be larger than length $b(a>b)$.

One of the most important differences of the proposed spatial SSM from common deployable scissor structures is the connection type between planar SLEs. In common deployable scissor structures, planar SLEs intersect at hinge points by means of a spatial intermediate connection element. Thus, the motion of one SLE directly affects the other SLEs of the system (Fig. 4). However, in the proposed SSM, planar SLEs or M-SLEs intersect at their pivot points by means of a properly configured intermediate connection element. Thus, SLEs of one plane can move without affecting the SLEs on perpendicular axes. This constitutes the main advantage of the proposed spatial SSM over existing designs.

As an example, a spatial translational SSM in space, which is composed of S-SLEs and is fixed at the location of intermediate connection element A, can be seen in Fig. 8. All other pivot nodes are restrained from vertical displacement but are free to slide horizontally. Because of the geometry and the connection type of the S-SLEs, this structural mechanism can change its length in $x$ or $y$ direction only, without affecting the length in the other direction. Thus, while mobility of common spatial SSMs (such as the SSM in Fig. 4 ) is equal to one, mobility of the system in Fig. 8 is equal to two. This affords the structure with increased transformability in comparison to existing designs.

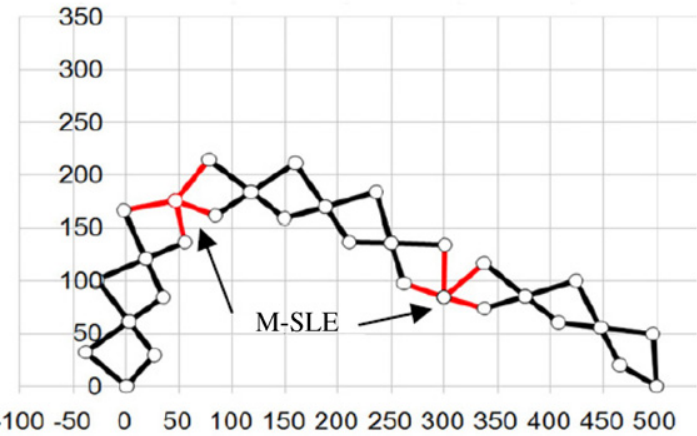

(b) Configuration 2.

(a) Configuration 1

Fig. 6. Contribution of M-SLEs to the transformation capability of a planar SSM.

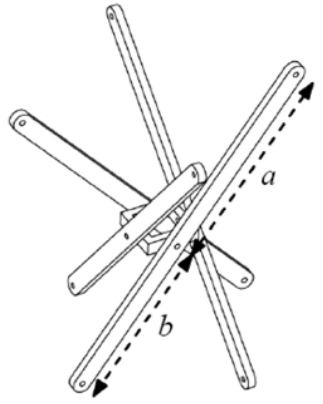

(a) S-SLE.

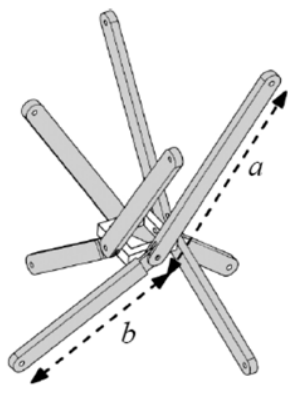

(b) MS-SLE.

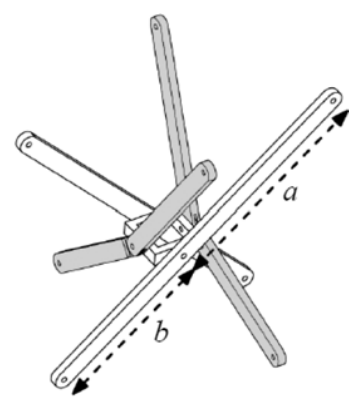

(c) HS-SLE.

Fig. 7. Primary elements of the proposed spatial SSM. 

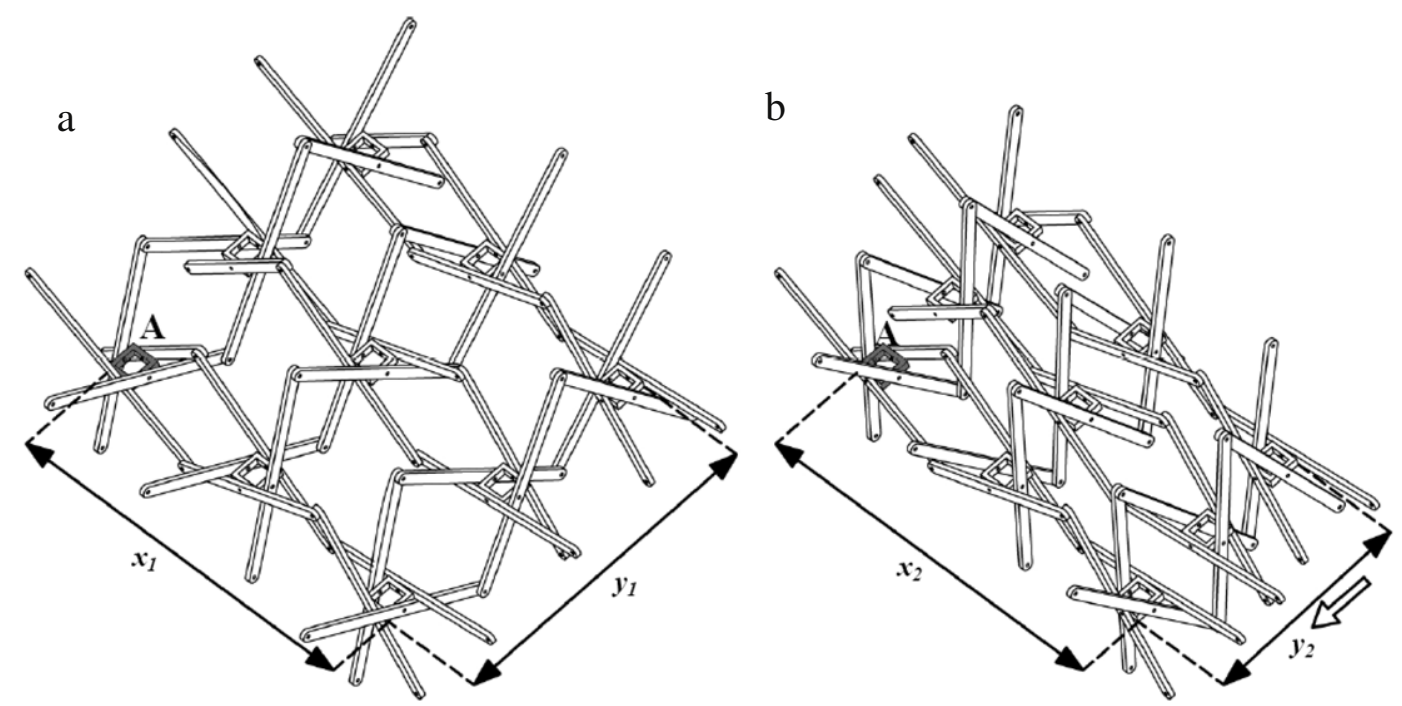

Fig. 8. Transformation of a spatial translational SSM composed of S-SLEs in $y$-direction only $\left(x_{2}=x_{1}, y_{2}<y_{1}\right)$.

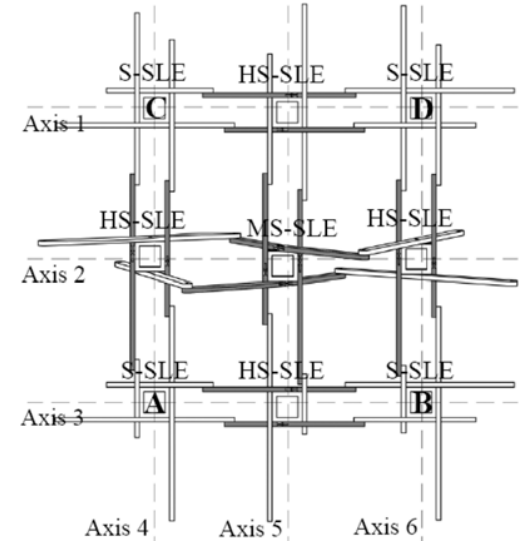

(a) Top view of configuration 1 .

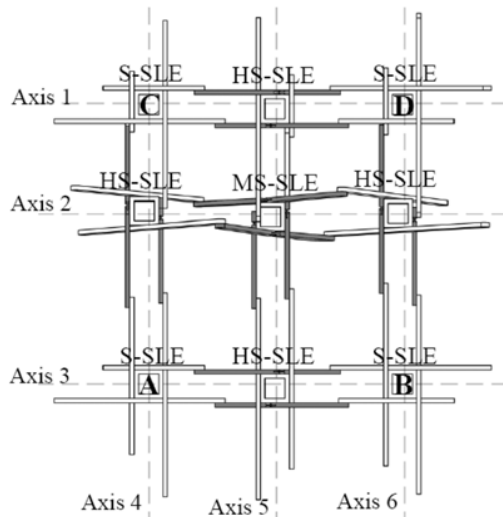

(b) Top view of configuration 2 .

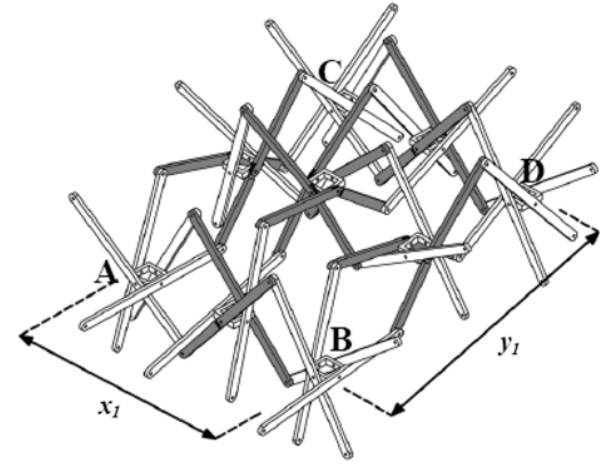

(c) Isometric view of configuration 1.

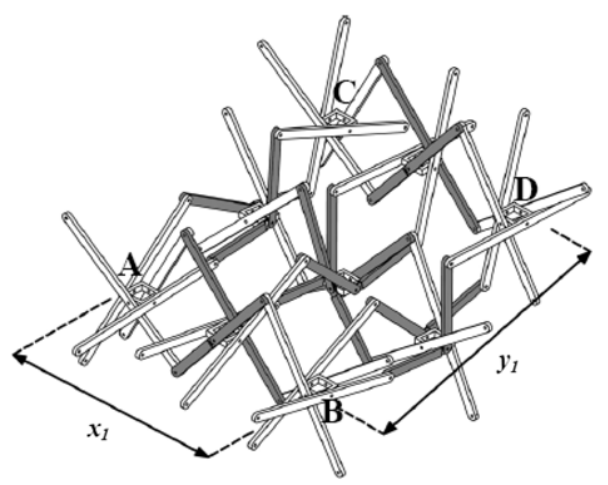

(d) Isometric view of configuration 2.

Fig. 9. Transformation of a curved SSM with one MS-SLE and four hybrid elements

Even though the S-SLE already provides partial improvement of form flexibility due to the connection between perpendicular SLEs at pivot points, the main contributions towards increased transformation capability are offered by the MS-SLE and the HS-SLE. To express this contribution, two different geometries of a transformable SSM are presented in Fig. 9. The system consists of four S-SLEs, four HS-SLEs and one MS-SLE, arranged in the middle. M-SLEs are emphasized as dark gray. Nodes A, B, C and D are hinged to rigid supports, so that the external dimensions of the SSM remain unchanged. Thus, mobility along each planar scissor axis is equal to two, and the struts in each separate axis constitute a planar transformable SSM of the type thoroughly explained in previous work of the authors [1].

While each planar SSM has mobility of two, the mobility of the whole transformable SSM is equal to one. Fig. 9(a) and (c) show top and isometric views of one typical configuration, respectively, while in Fig. 9(b) and (d) corresponding views of another configuration are illustrated. The primary elements are appropriately arranged, so that the structural mechanism can transform its shape without changing the outer dimensions of the 


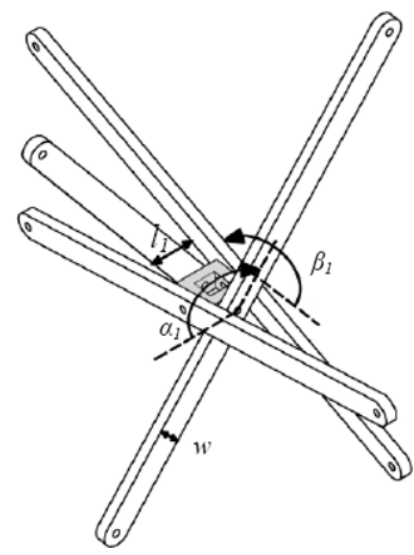

(a) Smaller connection element.

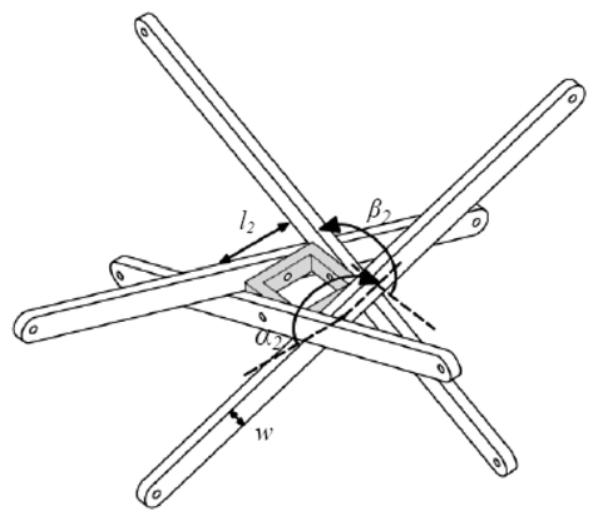

(b) Larger connection element.

Fig. 10. Transformation limits of the S-SLE.

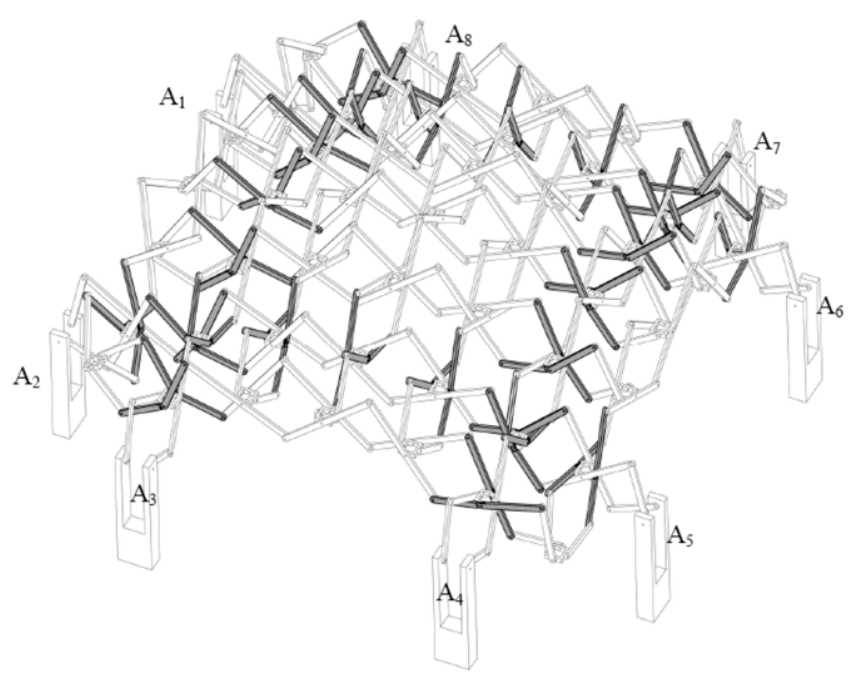

(a) Perspective view.

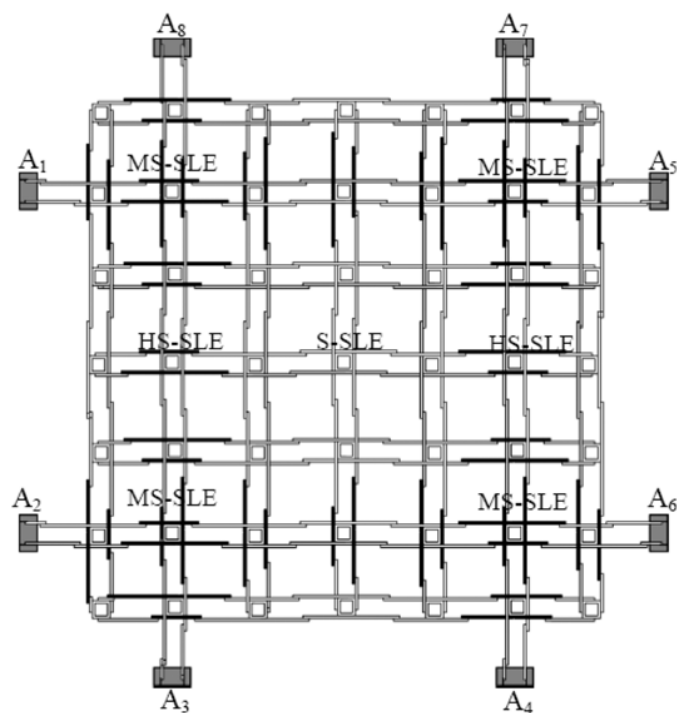

(b) Plan view.

Fig. 11. Proposed spatial SSM.

whole system and the size of the covered area. This constitutes an important advantage over common deployable structures, which cannot transform when they are restrained at two or more points.

\subsection{Geometric properties of primary elements}

The primary elements in Fig. 7 can be thought as the spatial extensions of SLEs and M-SLEs on perpendicular planes. Each strut of these elements can rotate about its pivot axis unless it collides with the strut in the perpendicular plane. This rotation capability is directly related to the dimensions of the connection element and of the struts in the perpendicular plane, as shown in Fig. 10. When the intermediate connection element becomes larger, the angles defining the rotation capability of the perpendicular struts increase as well: when $l_{2}>l_{1}$, then $\alpha_{2}>\alpha_{1} ; \beta_{2}>\beta_{1}$. Thus, the larger connection elements increase the transformation capability of the unit; however, they also increases the weight of the structure and introduce eccentricities, which are undesirable from a structural response point of view.

The transformation capability of the S-SLE depends also on the cross-section $(w)$ of the struts. When $w$ increases, the transformability range of the struts and angles $\alpha$ and $\beta$ decrease.

\section{Proposed spatial SSM and its geometric properties}

The proposed spatial SSM, presented in Fig. 11, has been derived from the primary elements in Fig. 7. Thus, the intersection between planar SLEs and M-SLEs in both directions is at the pivot point, while it is at the hinge points for common deployable structures. This structural mechanism is a scissor shell structure; and its superiority over the common deployable structures lies in its increased transformation capability. The proposed system can be transformed between various curved and double curved geometries, without changing the size of the covered area.

To generate the proposed spatial SSM in Fig. 11, 25 S-SLEs, 4 MS-SLEs, 20 HS-SLEs and 8 special SLEs for the support points have been utilized. The layout of these elements can be seen in Fig. 11(b). M-SLEs are again shown in dark gray. The dimensions of all primary elements are identical, and they are derived from the corresponding planar SLE and M-SLEs. The system is connected to the ground at eight points $\left(A_{1}, A_{2}, \ldots, A_{8}\right)$. The span of the system is $14 \mathrm{~m}$ in both directions; and the lengths of the struts are $270 \mathrm{~cm}$. Length $a$ and $b$ for the primary elements, described in Fig. 7, are $150 \mathrm{~cm}$ and $120 \mathrm{~cm}$ respectively.

As is also the case for common structural mechanisms, additional actuators are needed to fix and transform the proposed 


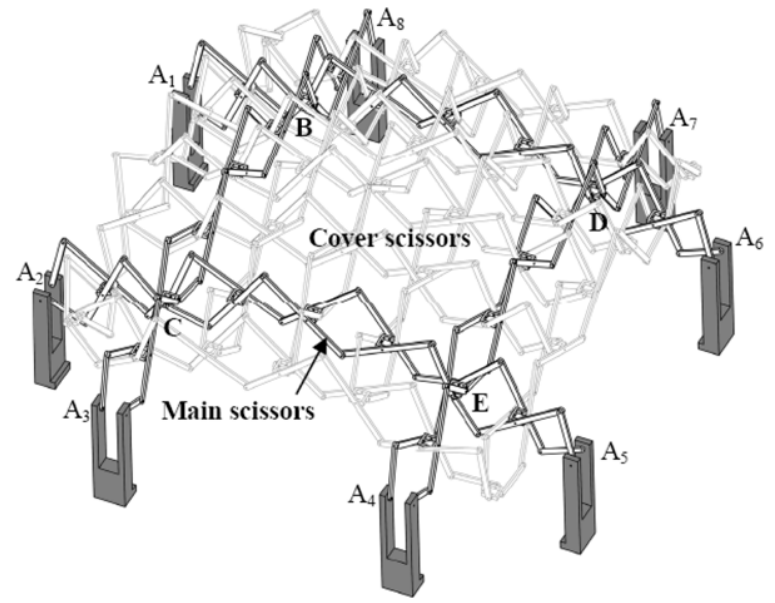

Fig. 12. Main scissors and cover scissors of the spatial SSM.

spatial SSM. From a wide range of experimental studies with small prototypes, it was concluded that these actuators should be utilized on the scissor axes connecting the support points $\left(A_{1}-A_{6}, A_{2}-A_{5}, A_{3}-A_{8}, A_{4}-A_{7}\right.$ axes). Thus, these four planar subSSMs become the main elements of the whole structure. The other elements which are called "cover scissors" are secondary members and do not have a significant role in form transformations. They only adapt themselves to the actual positions of the main scissors (see Fig. 12). Therefore, during the kinematic analysis of the proposed spatial structure, the transformation capability of the main SSMs was investigated thoroughly; but the transformation of the cover scissors was not taken into consideration.

In order to find the number of required actuators, the mobility of the structure should be calculated. To facilitate this calculation, the main scissors in Fig. 12 are abstracted and presented in Fig. 13. In this figure it can be seen that due to the use of revolute joints at the connections between the struts, all subsystems $\left(\mathrm{A}_{3} \mathrm{CBA}_{8}, \mathrm{~A}_{4} \mathrm{EDA}_{7}, \mathrm{~A}_{2} \mathrm{CEA}_{5}\right.$ and $\left.\mathrm{A}_{1} \mathrm{BDA}_{6}\right)$ cannot deviate from their planes, and can achieve only planar transformations. The subsystems intersect at certain points $(B, C, D$ and $E)$ which are called "knots". In order to maintain the planes of the main scissors, knots can only move through the $z$ direction. This property is a very important factor for the transformation capability of the proposed spatial SSM.

When the Freudenstein-Alizade formula is applied to find the mobility, it is seen that the abstracted system in Fig. 13 has 40 joints and 12 loops (such as $\mathrm{A}_{3} \mathrm{CG}, \mathrm{A}_{3} \mathrm{CEA}_{4}, \mathrm{~A}_{4} \mathrm{EI}$, etc.). All sub-systems are planar, so $\lambda$ is equal to three. According to these variables, the mobility of the system is equal to four $(M=4)$. This means that, theoretically, a minimum of four actuators are needed to control the geometry of the structural mechanism. Feasible locations of these actuators are discussed in Section 5.

The transformation capability of the whole structure is directly related to the transformation capability of the planar members. Some sample transformations of the proposed structural mechanism are illustrated in Fig. 14. From this figure, it can be understood that the proposed SSM can obtain various curved and double curved shapes without changing the boundaries of the covered space.

\section{Structural behavior of the proposed spatial SSM}

In order to understand the structural behavior of the proposed spatial SSM, and to obtain the most feasible locations for the needed actuators, a set of structural analyses have been carried out by imposing typical loading patterns in two different geometric configurations of the structure.

The system in Fig. 11 has been used in the static analysis. However, only the main scissors in Fig. 12 were taken into consideration, while the cover scissors were ignored in the analysis. This is because the cover scissors have a secondary role in transferring the loads to the support points through the main scissors, and can be studied independently. Thus, loads for the entire structure were applied directly on the main scissors.

Due to the relatively high flexibility, geometric nonlinearity has been taken into account in the analyses, while the material was assumed to be linear elastic, confirming this assumption later on by carrying out elastic checks for cross-sectional and member strength S275 steel with an elastic modulus equal to $21000 \mathrm{kN} / \mathrm{cm}^{2}$, Poisson's ratio equal to 0.3 and yield stress equal to $27.5 \mathrm{kN} / \mathrm{cm}^{2}$ was considered as the material of the struts. The analyses were performed with the finite element software ADINA. The model consisted of Hermitian beam elements with six degrees of freedom at each end, and was suitably discretized in order to obtain sufficient accuracy. In order to simplify the model eccentricities between struts were neglected, and all struts on each frame were assumed to be in the same plane.

Two sample geometric configurations (symmetric cross-vault shape and asymmetric shape) were modeled and analyzed. The response of the structure in these two geometries against vertical load, representing self weight and snow load has been simulated. All loads were applied as concentrated on the 12 nodes where cover scissors are supported on the main scissors. Due to the arched shape of cover scissors loads exerted by them on the main scissors have not only a vertical but also a horizontal component.

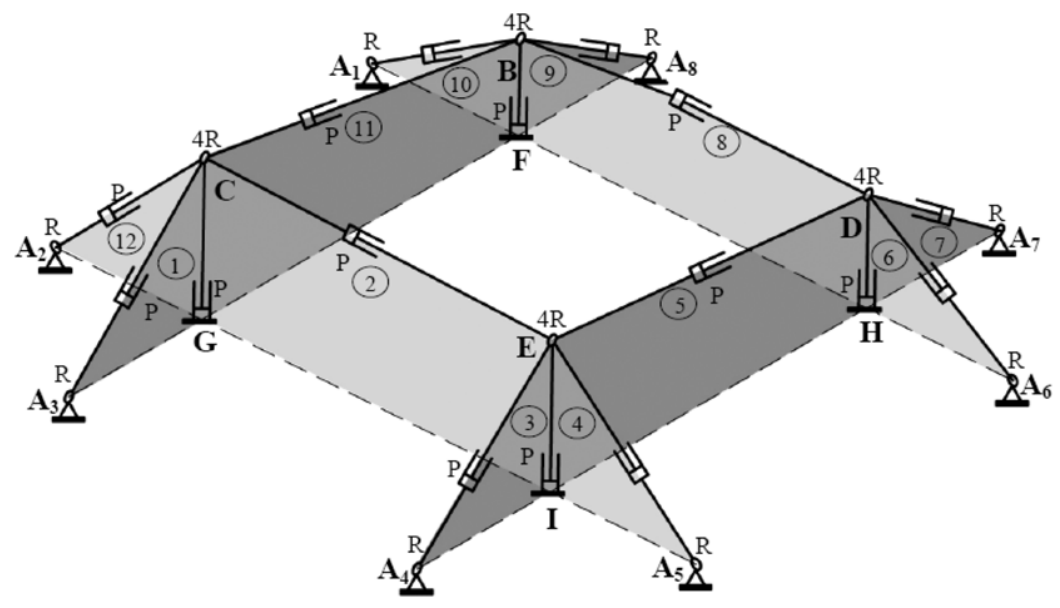

Fig. 13. Abstraction of the main scissors. 

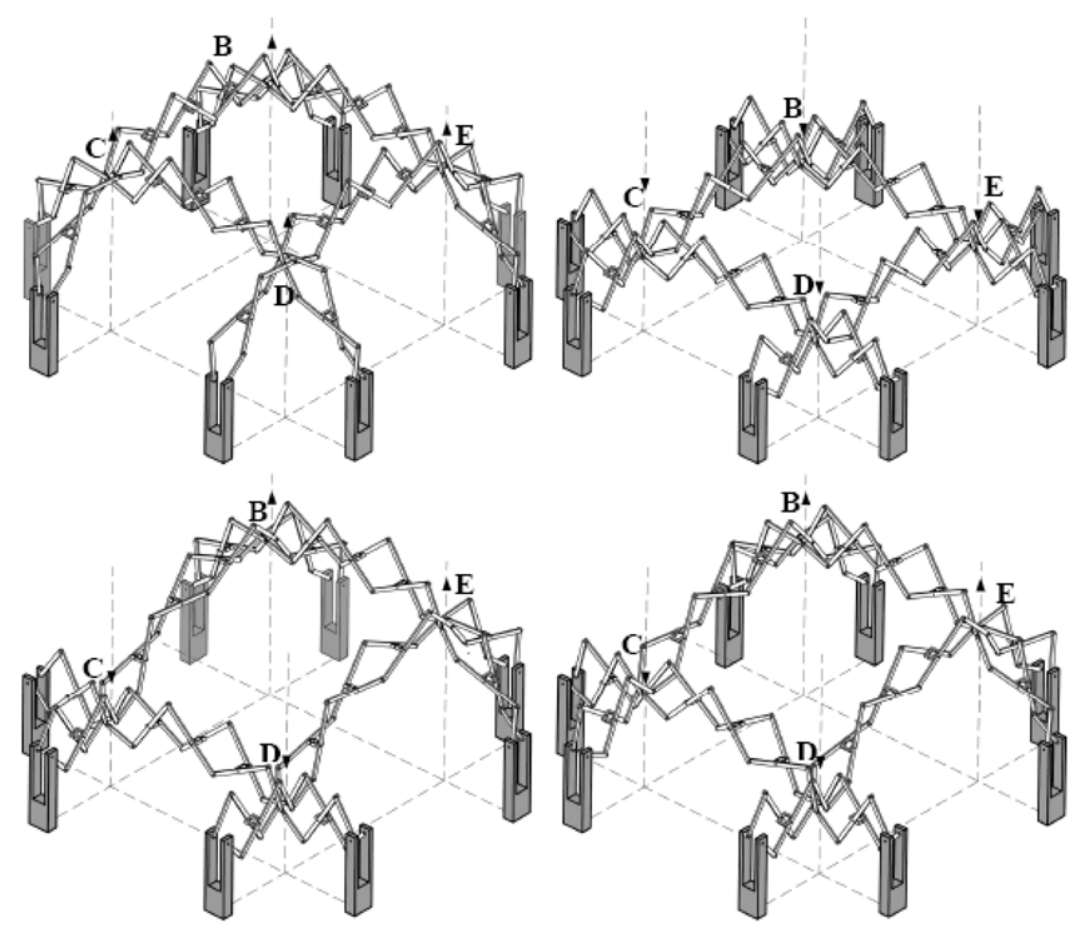

Fig. 14. Successive geometric configurations of the main scissors of the spatial SSM.

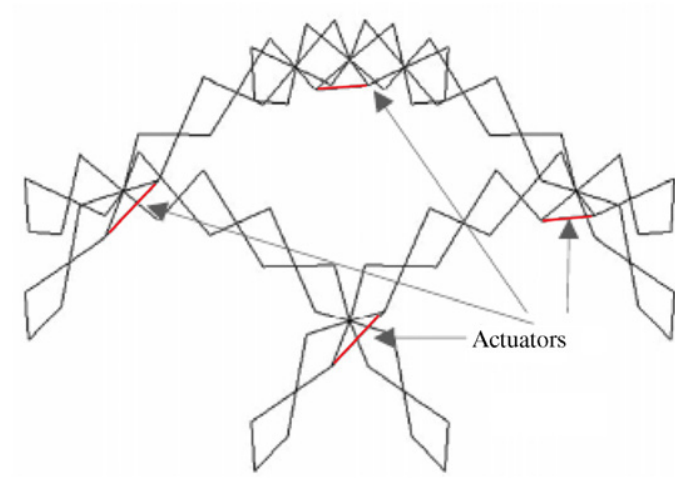

(a) Actuator configuration 1 .

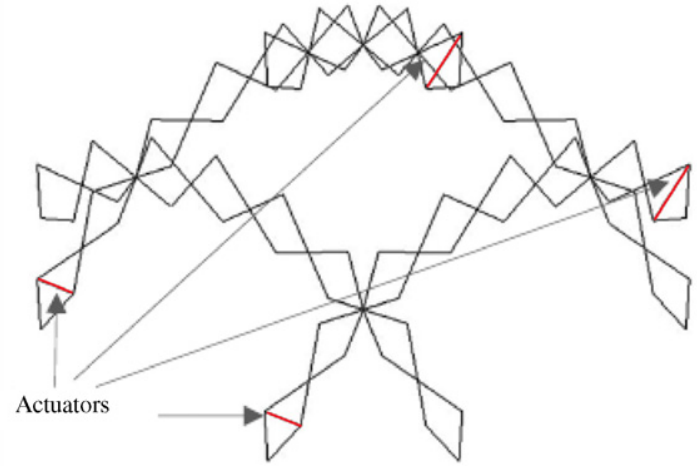

(b) Actuator configuration 2 .

Fig. 15. Two alternative actuator configurations for the solution with four actuators (red color).

The total vertical component applied on each of the 12 nodes was equal to $15 \mathrm{kN}$, while the horizontal component was equal to $2 \mathrm{kN}$.

Rectangular hollow cross-sections of $0.1 \mathrm{~m} \times 0.6 \mathrm{~m} \times 0.01 \mathrm{~m}$ with an area equal to $0.0136 \mathrm{~m}^{2}$ and a moment of inertia about the strong axis equal to $0.0005 \mathrm{~m}^{4}$ were employed for all members. Elastic strength checks of normal stresses due to axial force and bending moment were carried out. A deflection limit of span/200, equal to $7 \mathrm{~cm}$, was used for serviceability checks.

\subsection{Structural response at symmetric cross-vault shape}

First, the structure with symmetric cross-vault shape was analyzed. The structure consists of four main frames that are connected to the ground at eight points, as shown in Fig. 12. In addition, there are four connections between perpendicular main frames. As these connections are at pivot locations of the main frames, it is necessary for stability that out-of-plane and torsional rotations be restricted. The capability of these connections to sustain a moment at these positions is, however, questionable from a practical point of view. Nevertheless, in a first model it was assumed that the connections between perpendicular frames

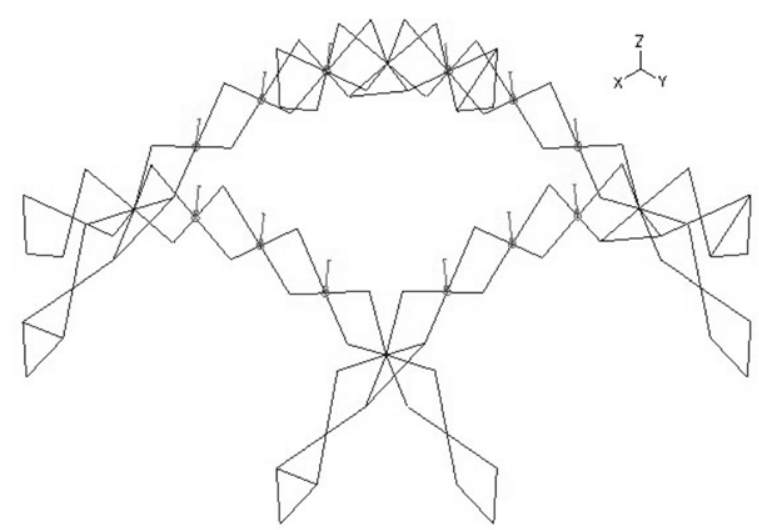

Fig. 16. Applied loads on the cross vault shaped structure with eight actuators.

are configured so that transfer of out-of-plane bending, as well as torsional moments was possible, while in-plane rotations were free. The ground supports were modeled in the same way. 


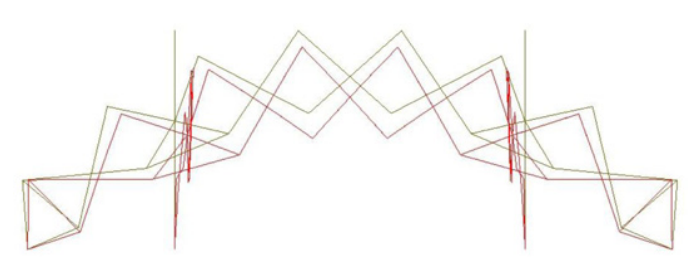

(a) In $X Z$ plane.

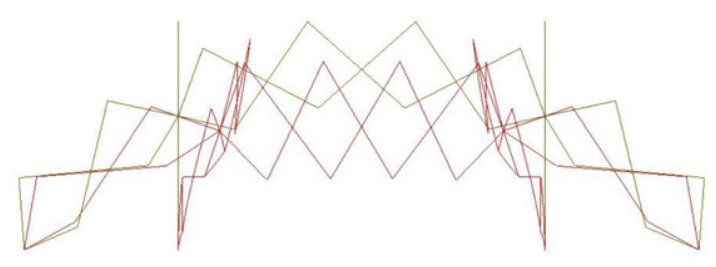

(b) In YZ plane.

Fig. 17. Undeformed (top configuration) and deformed (bottom configuration) shapes of the symmetric cross-vault shaped structure.

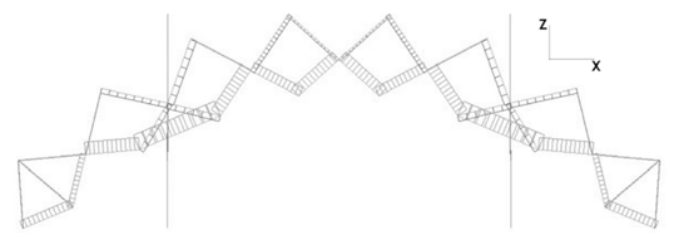

(a) In $X Z$ plane.

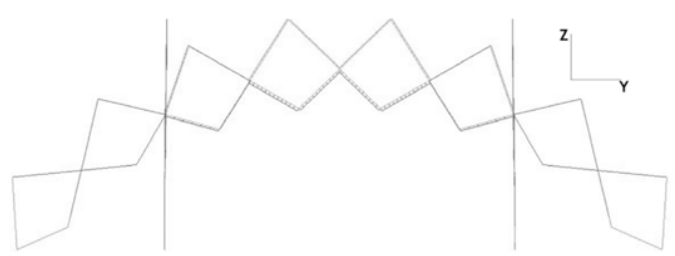

(b) In YZ plane.

Fig. 18. Axial force diagrams of the symmetric cross-vault shaped structure. The maximum axial force in the structure is equal to $233 \mathrm{kN}$.

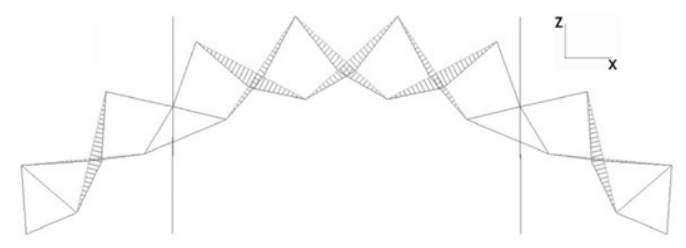

(a) In XZ plane.

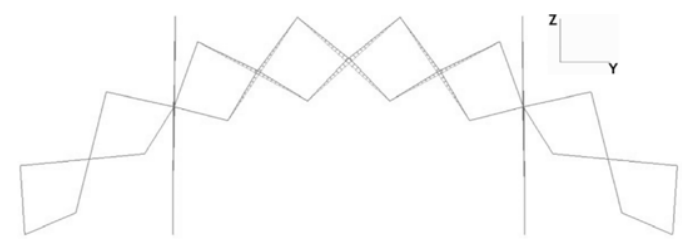

(b) In YZ plane.

Fig. 19. In-plane bending moment diagrams of the symmetric cross-vault shaped structure. The maximum in-plane bending moment in the structure is $136 \mathrm{kN}$.

Table 1

Maximum displacements of the symmetric cross-vault shaped structure.

\begin{tabular}{ll}
\hline Maximum displacement & Value $(\mathrm{cm})$ \\
\hline$Z$ (vertical) & 5.07 \\
$X$ & 1.38 \\
$Y$ & 5.15 \\
\hline
\end{tabular}

From the kinematic analysis, it is known that the mobility of the structure is equal to four; so four actuators are theoretically sufficient for stabilizing the structure. Linear elastic analysis was successfully carried out for validating this fact. The four actuators were symmetrically located in the frames of one direction to prove the stability of the whole structure with minimum number of actuators. Two alternative actuator configurations are shown in Fig. 15.

It should be also noted that for this analysis the actuators were considered to be "locked" in the final position of the structure, behaving in this "deployed phase" as common structural struts. The deployment process was not simulated, as in the "deployment phase" the structure behaves as a mechanism and no stresses develop, provided that the deployment rate is sufficiently small. Actuators were hence modeled using Hermitian beam elements having only the local torsional rotations restricted at their ends.

However, the results indicated that the structure with four actuators was too flexible for this span and level of loading. Moreover, increasing the number of actuators contributed a lot more to enhanced rigidity of the structure than increasing the cross-section's dimensions. Thus, eight actuators were finally used, placed as shown in Fig. 16. In this figure, applied loads can be seen as well.

Deformed shape, axial force and bending moment diagrams for the aforementioned load case are shown in Figs. 17-19, respectively. It is noted that the main frames equipped with actuators are much stiffer, thus they "attract" much larger forces.

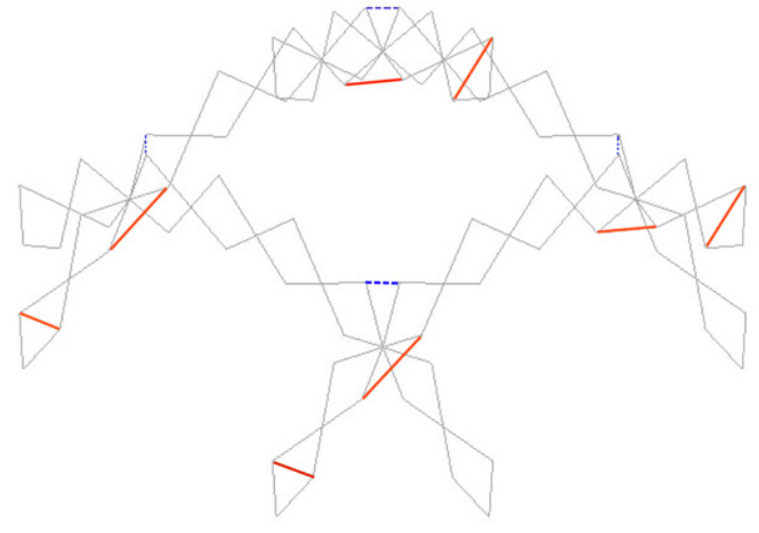

Fig. 20. Cross-vault shaped structure with eight actuators and four telescopic members (additional dotted lines).

The maximum displacements are given in Table 1 . The maximum vertical displacement allowed for reasons of serviceability is $7 \mathrm{~cm}$, thus serviceability checks are satisfied. The maximum normal stress due to combined axial force and biaxial bending moment is found to be approximately equal to $9 \mathrm{kN} / \mathrm{cm}^{2}$, much smaller than the yield stress $27.5 \mathrm{kN} / \mathrm{cm}^{2}$. Thus, serviceability governs the design.

As mentioned above, in reality it is questionable whether the four connections between perpendicular frames can be detailed in such a way that they are capable of resisting out-of-plane and torsional rotation. For this reason it was decided to configure these connections as hinges, thus to consider that the three rotational degrees of freedom of all members converging at each of these points are free and no moments are transmitted. However, as a result, the whole structure becomes a mechanism and additional members are needed in order to stabilize it. A simple solution was 


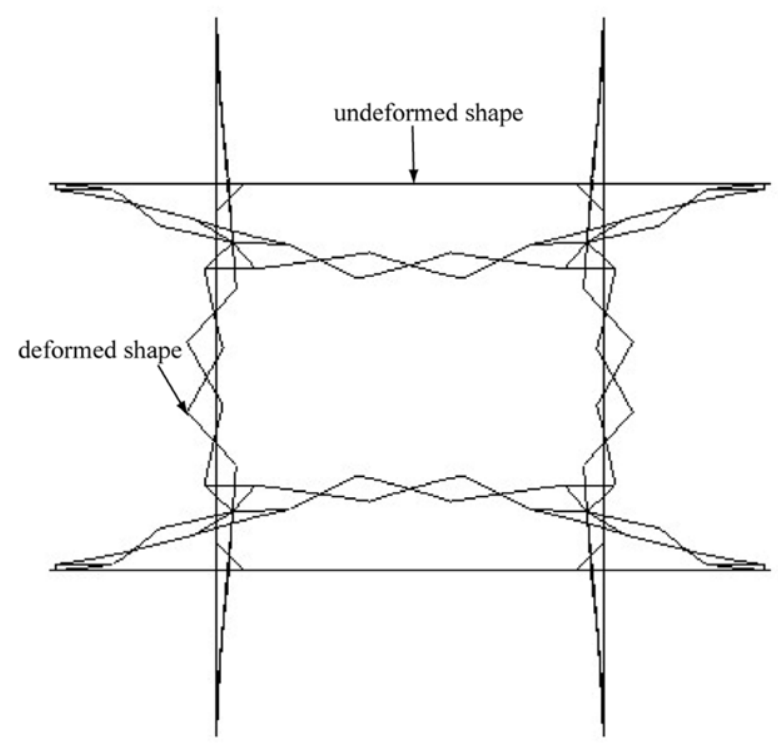

Fig. 21. Deformed and undeformed shapes of the symmetric cross-vault shaped structure with four telescopic members in plan.

adopted, providing additional members between main scissors, as shown in Fig. 20. These members can be telescopic, so that their length can be adapted to the required one based on the structure's overall geometry. It should be noted that these members are not actuators and that they are pinned at their ends. These additional members were modeled exactly in the same way as the actuators.

Applying the load pattern used for the previous model, the resulting deformation and bending moment diagrams are shown in Figs. 21 and 22, respectively.
Table 2

Maximum displacements of the symmetric cross-vault shaped structure with four telescopic members.

\begin{tabular}{ll}
\hline Maximum displacement & Value $(\mathrm{cm})$ \\
\hline$Z$ (vertical) & 5.90 \\
$X$ & 1.82 \\
$Y$ & 5.79 \\
\hline
\end{tabular}

The maximum displacements of the cross-vault structure with eight telescopic members are given in Table 2. Deformations are now a little larger but serviceability checks are satisfied. The maximum normal stress is now approximately equal to $10 \mathrm{kN} / \mathrm{cm}^{2}$, thus is still not critical.

\subsection{Structural response at asymmetric shape}

In the second phase of the analysis, each scissor frame has been transformed to a different geometry and the structure has now been transformed into an asymmetric shape. The number and locations of actuators and telescopic members, loading, material and cross-sections of the struts are exactly the same as in the symmetric cross-vault shape structure. Views of this structure in the $X Z$ and $Y Z$ planes are shown in Fig. 23, with maximum displacements given in Table 3, while bending moment diagrams are shown in Fig. 24. Vertical displacements are now smaller but significant horizontal deformations are introduced. The maximum normal stress is now found to be approximately equal to $22 \mathrm{kN} / \mathrm{cm}^{2}$, still smaller than the yield stress $27.5 \mathrm{kN} / \mathrm{cm}^{2}$ but now nearly critical.

From the analysis results important characteristics of the proposed spatial SSM can be understood. First of all, the proposed spatial SSM is stable at both symmetric cross-vault shape and asymmetric shapes, if four actuators, which are theoretically sufficient to stabilize the structure, are used at critical locations.

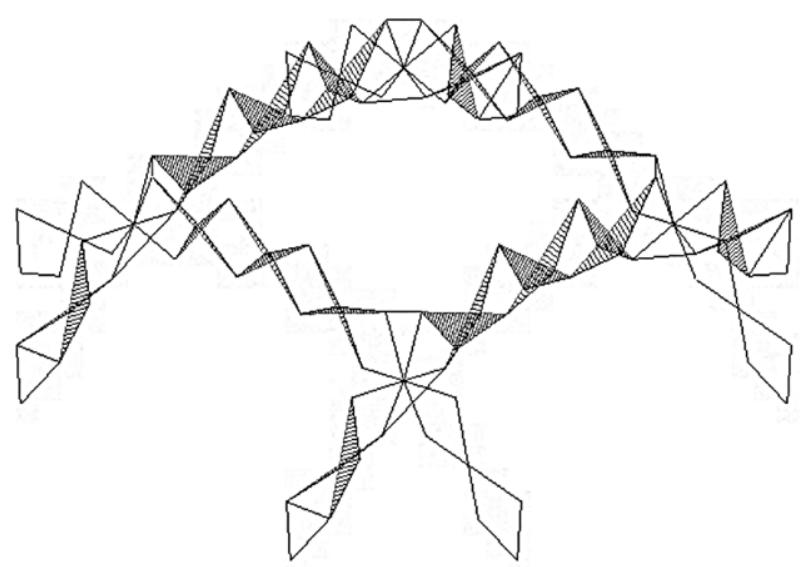

(a) In-plane bending moments for the whole structure with the maximum value being equal to $143 \mathrm{kN} \mathrm{m}$.

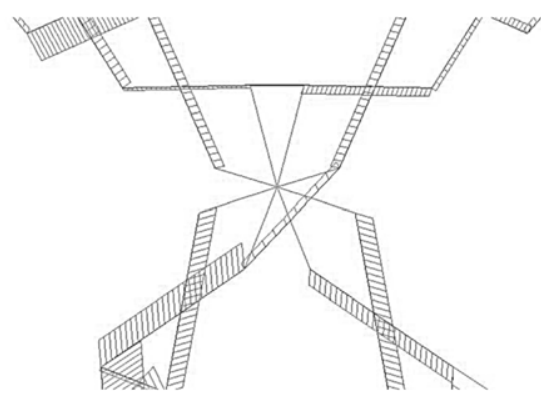

(b) Torsional moment (detail).

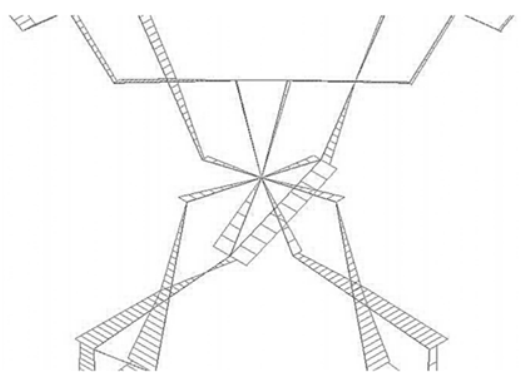

(c) Out-of-plane bending moment (detail).

Fig. 22. Moment diagrams of the symmetric cross-vault shaped structure with four telescopic members. 


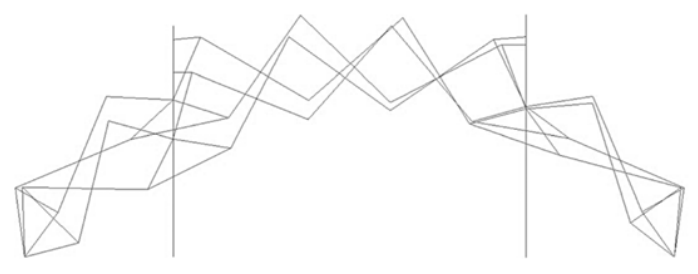

(a) In $X Z$ plane.

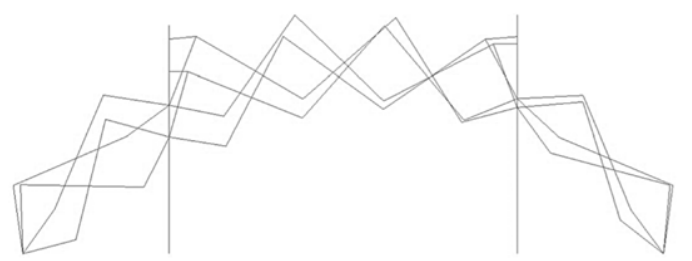

(b) In YZ plane.

Fig. 23. Views of the asymmetric structure with four telescopic members.

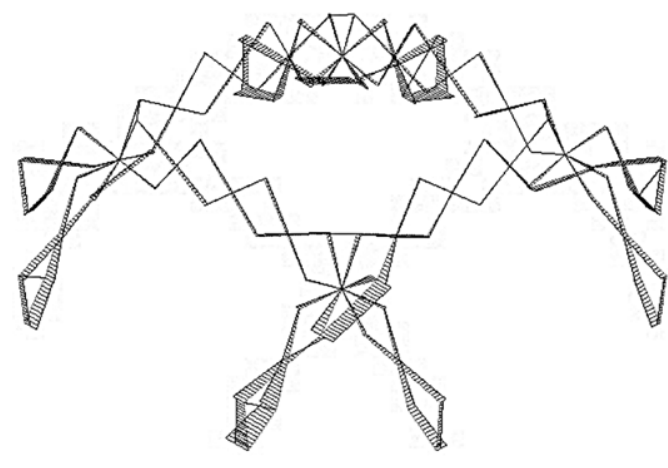

(a) Out of plane bending.

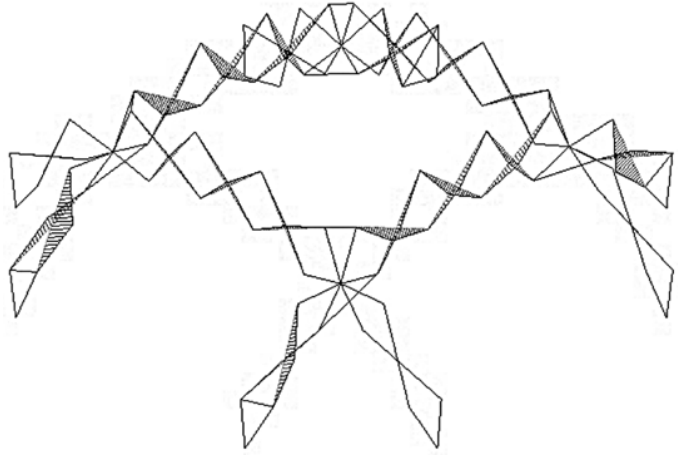

(b) In-plane bending.

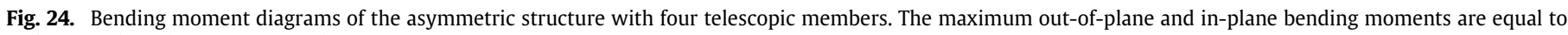
$26 \mathrm{kN} \mathrm{m}$ and $317 \mathrm{kN} \mathrm{m}$, respectively.

Table 3

Maximum displacements of the asymmetric structure with four telescopic members.

\begin{tabular}{ll}
\hline Maximum displacement & Value $(\mathrm{cm})$ \\
\hline$Z$ (vertical) & 4.74 \\
$X$ & 2.25 \\
$Y$ & 5.06 \\
\hline
\end{tabular}

However, addition of four more actuators than the necessary ones has been proved to be practically needed for stiffness in the specific structure analyzed. In addition, release of the bending moments in the three spatial directions at the four critical points of intersection of perpendicular frames is a more realistic approach as far as the behavior of the connections at these points is concerned. Additional telescopic members (not actuators) should then be used for stabilizing the structure. Further optimization could be possible by distributing the actuators in main scissors of both directions, varying the cross-sections between main scissors and/or along each main scissor.

\section{Summary and conclusions}

A novel analytical design and implementation framework for spatial scissor-hinge structural mechanisms has been proposed; and main geometric, kinematic and structural principles of this proposal have been introduced. The main differences of this novel structural mechanism are the new connection type and the proposed primary element called Modified Spatial ScissorLike Element (MS-SLE). Due to this novel element and the utilized connection type, the proposed structural mechanism can offer a wide range of form flexibility without changing the size of the covered area. Because of this superior property, the proposed spatial SSM can be utilized as permanent adaptive roof structure.

Geometric, kinematic and structural properties of the proposed SSM were thoroughly analyzed. The structure offers improved transformability with respect to common deployable examples, however, it needs more actuators to be moved and fixed. Moreover, increased transformability comes at the expense of reduced stiffness and strength. In the paper, potential number and location of these additional actuators were investigated. Different actuator locations and their effects on structural stability were tested. Consequently, the analyses showed that, by suitable number and positioning of actuators, structures of that type covering small to medium spans are indeed able to carry low to medium loads in a satisfactory manner.

\section{References}

[1] Söylemez E. Mechanisms. Ankara: METU Press; 1999.

[2] Chen Y. Design of structural mechanisms. Ph.D. thesis. Oxford: Department of Engineering Science, University of Oxford; 2003.

[3] Gantes CJ. Deployable structures: analysis and design. Boston: WIT Press; 2001.

[4] Zuk W, Clark R. Kinetic architecture. New York: Van Nostrand Reinhold; 1970.

[5] Chilton J. Space grid structures. Oxford: Architectural Press; 2000.

[6] Escrig F, Valcarcel JP. Geometry of expendable space structures. Int J Space Struct 1993;8:71-84.

[7] Escrig F. Expandable space frame structures. In: Nooshin H, editor. Proceeding of the 3rd international conference on space structures. Guildford (UK): Elsevier Applied Science Publishers; 1984. p. 845-50.

[8] Escrig F. Expendable space structures. Space Struct J 1985;2(1):79-91.

[9] Escrig F, Valcarcel JP. Curved expandable space grids. In: Topping BHV, editor. Proceedings of the international conference on the design and construction of non-conventional structures. London (England): Civil-Comp. Press; 1987. p. 157-68.

[10] Escrig F, Valcarcel JP. Great size umbrellas with expendable bar structures. In: Proceeding of the 1st international conference on lightweight structures in architecture. 1986 (1). p. 676-81.

[11] Calatrava S. Zur faltbarkeit von fachwerken. Ph.D. thesis. Zurich: ETH; 1981.

[12] Tischhauser A, von Moos S. Calatrava: public buildings. Basel (Switzerland): Birkhauser Press; 1998.

[13] Hoberman C. Unfolding architecture: an object that is identically a structure and a mechanism. Archit Design 1993;63:56-9.

[14] Al Khayer M, Lalvani H. Scissors-action deployables based on space-filling of polygonal hyperboloids. In: Pellegrino S, Guest SD, editors. Proceedings of IUTAM IASS symposium on deployable structures: theory and applications. Cambridge: Kluwer Academic Publishers; 1998. p. 1-10.

[15] Dieguez RG, Cozar JCG. Florin system double layer spatial deployable structures with frames of rhombuses and scissors. In: Pellegrino S, Guest SD, editors. Proceedings of IUTAM IASS symposium on deployable structures: theory and applications. Cambridge: Kluwer Academic Publishers; 1998. p. $117-26$. 
[16] Escrig F, Valcarcel JP. Analysis of expendable space bar structures. In: Heki K, editor. Proceeding of IASS symposium on shells, membranes, and space frames, vol. 3. Osaka (Japan): Elsevier Science Publishers; 1986. p. 269-76.

[17] Gantes C, Konitopoulou E. Geometric design of arbitrarily curved bi-stable deployable arches with discrete joint size. Internat J Solids Structures 2004; 41:5517-40.

[18] Gantes CJ, Connor JJ, Logcher RD. A systematic design methodology for deployable structures. Int J Space Struct 1994;2(9):67-86.

[19] Gantes C, Logcher RD, Connor JJ, Rosenfeld Y. Deployability conditions for curved and flat, polygonal and trapezoidal deployable structures. Int J Space Struct 1993;8:97-106.

[20] Langbecker T. Kinematic analysis of deployable scissor structures. Int J Space Struct 1999;14:1-15.

[21] Langbecker T, Albermani F. Kinematic and non-linear analysis of foldable barrel vaults. Eng Struct 2001;23:158-71.

[22] Zhao JS, Feng ZJ. The mechanism theory and application of deployable structures. Mech Mach Theory 2009;44:324-35.
23] Gan WW, Pellegrino S. Closed-loop deployable structures. In: Proceedings of 44th AIAA/ASME/ASCE/AHS/ASC structures, structural dynamics and materials conference. 2003.

[24] Gan W, Pellegrino S. Numerical approach to the kinematic analysis of deployable structures forming a closed loop. In: Proceedings of the IMECH E part $C$ journal of mechanical engineering science. Professional Engineering Publishing; 2006. p. 1045-56.

[25] You Z, Pellegrino S. Foldable bar structures. Internat J Solids Structures 1997; 15(34):1825-47.

[26] Akgün Y, Gantes CJ, Kalochairetis K, Kiper G. A novel concept of convertible roofs with high transformability consisting of planar scissor-hinge structures. Eng Struct 2010;32:2873-83.

[27] Norton RL. Design of machinery: an introduction to the synthesis and analysis of mechanisms and machines. 3rd ed. Singapore: McGraw Hill Education Press; 2004 (International Edition).

[28] Freudenstein F, Alizade R. On the degree of freedom of mechanisms with variable general constraint. In: Proceedings of IFToMM IV world congress. 1975. p. 51-6. 